\title{
The Neurotrophic and Anti-Inflammatory Effect of $\beta$ - asarone on the DA-Induced the Pathology of Minimal Hepatic Encephalopathy
}

\author{
Jian wang \\ Wenzhou Medical University \\ Xuebao Wang \\ Wenzhou Medical University \\ Leping Liu \\ Wenzhou Medical University \\ $\mathrm{He} \mathrm{Yu}$ \\ Wenzhou Medical University \\ Ruimin You \\ Wenzhou Medical University \\ Xiaoai Lu \\ Wenzhou Medical University \\ Saidan Ding ( $\nabla$ firstdsdan@hotmail.com ) \\ Wenzhou Medical University
}

\section{Research}

Keywords: minimal hepatic encephalopathy (MHE), Dopamine (DA), tumor necrosis factor-a (TNFa), glial cell line-derived neurotrophic factor (GDNF), Nerve growth factor (NGF), $\beta$-asarone (BASA)

Posted Date: September 2nd, 2020

DOI: https://doi.org/10.21203/rs.3.rs-69260/v1

License: (c) (1) This work is licensed under a Creative Commons Attribution 4.0 International License. Read Full License 


\section{Abstract}

Background: The mechanism underlying the impaired cognitive function and memory loss in Minimal hepatic encephalopathy (MHE) remains unclear. Dopamine (DA) is reported to be associated with dementia.

Methods: In this study, we investigated mechanism underlying DA-induced MHE pathology by immunoblotting, ELISA, FM4-64 and fluorescence staining.

Results: We observed that MHE brains showed the increased content of DA, after administration of antiDA antibody, and cognitive loss in MHE rats was recovered to the normal level, indicating the involvement of DA in the pathogenesis of MHE. Moreover, DA $(10 \mu \mathrm{M})$ treatment obviously induced the decrease in the production of GDNF/NGF and the increase in TNFa levels in primary cultured neurons, which were blocked by addition of $\beta$-asarone ( $\beta A S A)$. We also demonstrated that DA stimulated the activation of ASK1/JNK1 pathway. and the addition of anti-TNFa antibody reversed the inactivation of Notch signaling, the downregulation of neurotrophins and synaptic loss.

Conclusions: Overall, we suggested that DA stimulated abundant production and secretion of neuronal TNFa, which elicited progressive loss of neurotrophic factors, leading to cognitive disorder of MHE.

\section{Introduction}

Brain dopamine (DA) has been implicated in cognitive processes such as working memory and cognitive flexibility [1] and the DA system is involved in cognitive decline [2]. Our previous studies have confirmed that the pathogenesis of MHE was caused by the abnormal elevation of intracranial DA $[3,4,5,6,7,8]$. It has been demonstrated as a novel regulatory mechanism that DA -induced astrocytic TNF-a release triggers progressive neurodegeneration, which leads to learning and memory decline in MHE [9]. Tumor necrosis factor-a (TNFa) signaling has been shown to regulate neuroinflammation, and plays roles in modulation of microglial activation [10], control of glutamatergic transmission and regulation of synaptic strength [11] in the central nervous system (CNS) [12]. Ample evidence suggests that elevated levels of TNFa have been implicated in some neurodegenerative disorders including Alzheimer's disease [13], Parkinson's disease [14] and HIV-associated dementia [15]. Hence, Neuroinflammatory conditions are often related with memory dysfunction $[16,17,18]$,which suggests that DA may be influenced on brain function involved with inflammatory factor. We further proposed that DA may regulate synaptic formation underlying long-term cognition with involvement of TNFa in MHE. Therefore, we hypothesized that the induction of cognitive decline by accumulation of DA levels in the MHE rats is associated with its ability to produce TNFa.

Here, we investigated (1) whether neuroinflammation was induced by DA in neurons in MHE; (2) whether neurons interference in disruption of synaptic formation triggered by DA in MHE. Moreover, $\beta$-asarone increased the expression of neurotrophin such as brain-derived neurotrophic factor (BDNF), glial cellderived neurotrophic factor, and ciliary neurotrophic factor in RSC96 cells [19]. $\beta$-asarone treatment also 
improved the spatial learning and memory in $A \beta$ (1-42)-induced memory-impaired rats [20]; in transgenic model of Alzheimer's disease such as Senescence-accelerated prone-8 (SAMP-8) mice [21]; and AßPP/PS1 double transgenic mice [22, 23],also in chronic exogenous corticosterone-induced memoryimpaired rats $[24,25]$. Then, we describe that the attribution of pathogenesis of MHE is upregulation of DA, followed by the production of TNFa from neurons, leading to the disruption of synaptic formation, which was diminished by $\beta A S A$ treatment.

\section{Materials And Methods}

\subsection{MHE models and treatment}

A total of 40 Sprague-Dawley (SD) rats (220-250 g) were used, and all guidelines of the care and use of animals for experimental procedures were laid down by the Ethics Committees of the Affiliated Hospital of Wenzhou Medical University regarding [26].

Before experimenting, all animals were subjected to behavioural tests: Y-maze (YM) and water-finding task (WFT). Normal values of YM and WFT these behavioural tests were obtained. Rats were then intraperitoneally (i.p.) injected with TAA (200 mg/kg in normal saline, Sigma-aldrich) to induce liver cirrhosis twice per week for 8 weeks. TAA-treated rats with symptoms of decreased movement, lethargy, and coma were diagnosed as HE. Then behavioral tests were conducted for TAA-treated rats with no HE symptoms. If values of YM were decreased or values of WFT were increased compared to mean \pm 1.96.SD of normal values, rats were included in the MHE group.

MHE Rats were anaesthetized with pentobarbital ( $40 \mathrm{mg} / \mathrm{kg}$, i.p.) and subjected to stereotaxic surgery. Two 23-gauge, stainless-steel, thin-wall cannulae were implanted to the right lateral ventricles at the following coordinates: $0.4 \mathrm{~mm}$ posterior to the bregma, $0.8 \mathrm{~mm}$ lateral to the midline, and $2.0 \mathrm{~mm}$ from the surface of the skull. MHE rats were injected with DA $(10 \mu \mathrm{g} / 3 \mu \mathrm{l}) 3$ times per week for 1 week through guide cannula, following by the intraperitoneal injection with $\beta$-asarone ( $\beta A S A, 20 \mu \mathrm{g}) 3$ times per week for 1 week. Then Rats were conducted with a YM and a WFT test.

\subsection{Behavioral test}

Rats were individually placed at the end of one of arm of the three arms in the apparatus for $\mathrm{YM}$, and were allowed to find the arm entries freely for $8 \mathrm{~min}$. Total arm entries and spontaneous alternation percentage (SA\%, a ratio of successful choices to total choices minus two) were measured [27].

Rats were deprived of water for $24 \mathrm{~h}$ and individually placed at the near-right corner of the testing apparatus for WFT and allowed to explore the tube and drink the water in the cubic alcove freely for $3 \mathrm{~min}$. The latency times of the first entry into the alcove (entry latency, EL), of the first touching/sniffing/licking of the water tube (contacting latency, $C L$ ) and of the initiation of drinking from the water tube (drinking latency, DL) were measured [28]. 


\subsection{Primary rats neurons culture and treatments}

Primary hippocampal rats neurons (PHNs) were obtained from freshly dissected hippocampus and cerebral cortex from 1-day-old SD rat pups by mechanical disruption in the presence of trypsin and DNase and then seeded in poly-L-lysine-precoated six-well plates at a density of $2 \times 10^{6}$ cells/well in Neurobasal ${ }^{\circledR}$ Medium (1X) supplemented with 0.5 mM GlutaMAX ${ }^{T M}-I, B-27 \AA$. Then neurons remained untreated or were stimulated with DA $(1,10$ or $20 \mu \mathrm{mol} / \mathrm{l})$ alone or after preincubation with JNK1 inhibitor SP600125, ASK1 inhibitor K811, $50 \mu \mathrm{M}$ Notch inhibitor MK-0752, $100 \mathrm{ng} / \mathrm{ml}$ Notch activator Jagged-1 or anti-TNFa antibody (ATAB), or treated with $\beta A S A$ in the presence or absence of DA together with MK0752/TNFa.

\subsection{PC12 cells culture and treatments}

The rat pheochromocytoma PC12 cell line were achieved from the American Type Culture Collection (ATCC, Manassas, VA). PC12 cells were routinely cultured in DMEM with $2 \mathrm{mM}$ glutamine, $10 \%$ horse serum, and $5 \%$ heat-inactivated fetal bovine serum (FBS) and passaged one time per week. Then PC12 cells remained untreated or were stimulated with DA $(1,10$ or $20 \mu \mathrm{mol} / \mathrm{l})$ alone or after preincubation with JNK1 inhibitor SP600125, ASK1 inhibitor K811, $50 \mu \mathrm{M}$ Notch inhibitor MK-0752, 100 ng/ml Notch activator Jagged-1 or anti-TNFa antibody (ATAB), or treated with $\beta A S A$ in the presence or absence of DA together with MK-0752/TNFa.

\subsection{Transfection for PC12 cells}

PC12 cells were transfected with Notch1/JNK1/ASK1/TNFa/ the Silencer Negative Control siRNA (Scrambled siRNA) (Santa Cruz, CA, USA) or Notch cDNA (activated) /empty vector plasmid (Millipore) using Lipofectamine 2000 (Invitrogen, Carlsbad, USA) according to the instructions suggested by the manufacture.

\subsection{Measurement of TNFa release}

TNFa level in the culture medium of neurons was measured using enzyme-linked immunosorbent assay (ELISA) kit (R\&D systems) in the light of the manufacturer's protocols and a Thermo-Fisher Multiskan MCC plate reader.

\subsection{Immunoblotting (IB) analysis}

The total amount of protein in the lysates were separated by $10 \%$ SDS-PAGE and electroblotted to PVDF membrane, the membrane was blocked with $5 \%$ non-fat dry milk dissolved in TBS-T (150 mM NaCl, $50 \mathrm{mM}$ Tris, $0.05 \%$ Tween 20 ), and incubated with following primary antibodies: DA, TNFa, GDNF, NGF, 
Apoptosis signal-regulating kinase1 (ASK1), c-Jun N-Terminal Kinase1 (JNK1), pASK1, pJNK1, Notch Intracellular Domain1 (NICD1), Hairy and enhancer of split1 (HES1), Homer, synaptotagmin, $\beta$-actin (Abcam) overnight at $4^{\circ} \mathrm{C}$, then probed with horseradish peroxidase-conjugated secondary antibody for $1 \mathrm{~h}$. After extensive washing, membrane was visualized by ECL reagent (Thermo) and exposed on Kodak BioMax film (Kodak). Intensities of protein bands were analysed with Quantity One software. Densitometry was indicated as ratio of proteins to $\beta$-actin levels.

\subsection{Functional labeling of presynaptic boutons with FM4-64}

FM4-64 staining (Invitrogen) was followed by the manufacturer's instructions. Briefly, neurons were incubated with $5 \mathrm{mg} / \mathrm{ml} \mathrm{FM4-64}$ and $50 \mathrm{mM} \mathrm{KCl-contained} \mathrm{Hank's} \mathrm{balanced} \mathrm{salt} \mathrm{solution} \mathrm{for} 1$ minute at $4{ }^{\circ} \mathrm{C}$.

\subsection{Double-labeled fluorescent staining}

Frozen brain sections or neurons cultured on glass coverslips were fixed with $4 \%$ paraformaldehyde for 30 min and treated with $0.1 \%$ Triton X-100 for 10 min, then blocked with bovine serum albumin (BSA) for $1 \mathrm{~h}$. Sections were then incubated with the following primary antibodies: DA, TNFa, GDNF, NGF, pASK1, pJNK1, NICD1, HES1, MAP2 (Abcam) overnight at $4{ }^{\circ} \mathrm{C}$, following with FITC (green)/Alexa Fluor 594 (red) conjugated secondary antibody for 30 min. A Leica TCS SP2 confocal laser scanning microscope was used for imaging.

\subsection{Dendritic spine density analysis in primary neurons}

After fixation, neurons cultured on glass coverslips were incubated with primary antibodies: microtubuleassociated protein 2B (MAP2B; 1:200; BD Transduction Laboratories, San Jose, CA, USA), and vesicular glutamate transporter 1 (vGlut1; 1:100; Neuromab, Davis, CA, USA), then with 1-hour secondary antibodyconjugated AlexaFluor (1:500; Life Technologies, Waltham, MA, USA). A z stack of optical section was imaged on a confocal laser scanning microscope (FV10i-W, Olympus, Japan).

\subsection{Statistical analysis}

All of the data were expressed as mean \pm SD. Data comparisons were analyzed using one-way analysis of variance (ANOVA). All of the data were detected in normal distribution and equal variances, when Back-ofthe-envelope test was used to verify the normal distribution, and F-test was applied for determining the equality of variances. Dunnett's post hoc multiple comparison test was applied when significant differences were detected by the ANOVA model. Then $\mathrm{P}$ values were made for adjustment by Bonferroni correction. The level of significance was determined for $P<0.05$ or $P<0.01$. All analyses were performed with SPSS 18.0 (PASW Statistics 18.0).

\section{Results}




\subsection{DA inhibits the production of neurotrophic factors in neurons}

First, we assessed the effect of DA on the production of neurotrophic factors in neurons. As indicated by the IB analysis, the decreased trend on the expressions of glial cell line-derived neurotrophic factor (GDNF)/Nerve growth factor (NGF) were observed after lower DA treatment, higher DA treatment significantly decreased the two proteins levels in PHNs (Fig. 1A and B). PC12 cells in response to DA showed that the expressions of GDNF/NGF were also reduced in a dose-dependent fashion (Fig. 1C and D). Time course analyses via IB analysis of PHNs treated with high dose of DA revealed steady decreases in GDNF/NGF expression up to $48 \mathrm{~h}$ (Fig. 1E and F). Double IF staining of PHNs using GDNF antibody combined with MAP2 antibody confirmed that high DA treatment induced the weak expression of GDNF (Fig. 1G). As analyzed by immunostaining, PC12 in response to DA displayed significant decrease in NGF expression (Fig. 1H). These results suggested that DA dramatically triggered the loss of neurotrophins.

\subsection{DA stimulated TNFa secretion from neurons}

Recent studies have reported that DA is involved in the process of inflammation [29, 30, 31, 32]. Therefore, we examined the effect of DA on the production of proinflammatory factor TNFa in neurons. Significant upregulation of TNFa was induced by higher DA treatment, but not by lower DA treatment in PHNs using IB analysis (Fig. 2A and B). Otherwise, IB analysis of PC12 cells showed that DA also significantly triggered TNFa expression in a dose-dependent manner (Fig. $2 \mathrm{C}$ and D). As determined by ELISA, DA significantly stimulated the release of TNFa with dose-dependence in PHNs (Fig. 2E). Parallelly, as shown by IB analysis, PHNs exhibited high TNFa levels in incubation of DA with a time-dependent manner (Fig. 2F and G). By ELISA, DA significantly elevated release of TNFa with time-dependence from PHNs (Fig. 2H). Double IF staining using TNFa antibody with MAP2 antibody confirmed that the expression of TNFa was obviously increased in PHNs after DA treatment (Fig. 2I). Immunostaining of PC12 cells in response to DA displayed the strong expression of TNFa (Fig. 2J). These results suggested that DA induced the production of proinflammatory factor TNFa in neurons in vitro.

\subsection{DA modulates production of TNFa by ASK1/JNK1 pathway in neurons}

In this experiment, we studied the mechanism underlying DA-induced TNFa production. ASK1 upregulates c-Jun N-terminal kinase (JNK1) signaling [33]. Inflammatory responses are frequently associated with cJun NH2-terminal kinase (JNK1) signaling pathway [34]. Thus, we examined the association of DAinduced production of TNFa with ASK1/JNK1 pathway in neurons. As determined by IB analysis, the phosphorylation of ASK1 was significantly increased in PHNs in response to DA with dose-dependent manner (Fig. 3A and B). DA treatment significantly elevated the phosphorylation of JNK1 in PHNs, which was reversed by ASK1 inhibitor K811 by IB analysis (Fig. 3C and D). DA-mediated the increase in TNFa expression in PHNs was significantly diminished by addition of K811 or JNK1 inhibitor SP600125 by IB analysis (Fig. 3E and F). The efficiency of ASK1 (Fig. 3G) or JNK1 siRNA (Fig. 3H) plasmid transfection 
was confirmed by qPCR in PC12 cells. Then we found that the increase in TNFa expression in response to DA were significantly diminished by ASK1 or JNK1 silencing in PC12 cells by IB analysis (Fig. $3 \mathrm{I}$ and J). ELISA of supernatants from PC12 cells exhibited that DA-induced release of TNFa was blocked by knockdown of ASK1 or JNK1 (Fig. 3K).

\subsection{DA induced downregulation of neurotrophins depending on TNFa in neurons}

In this experiment, we tried to confirm whether DA-induced synaptic loss was associated with ASK1/JNK1/TNFa pathway. Parallelly, as shown by IB analysis, PHNs exhibited lower GDNF/NGF levels after DA treatment, addition of K811, SP600125 or ATAB blocked the decrease in GDNF/NGF expression (Fig. 4A and B). The efficiency of TNFa siRNA plasmid transfection into PC12 cells was confirmed by qPCR (Fig. 4C). Parallelly, as shown by IB analysis, PC12 cells exhibited the increase in GDNF/NGF levels after knockdown of ASK1/JNK1/TNFa (Fig. 4D and E). Furthermore, by IB analysis, exposure of PHNs to DA also displayed the decreased expression of Homer/synaptotagmin which were blocked by addition of K811, SP600125 or ATAB (Fig. 4F and G). Furthermore, by immunostaining, GDNF expression in PC12 cells with DA treatment was markedly decreased, while K811, SP600125 or ATAB significantly increased the protein expression (Fig. 4H). Double IF staining of PHNs with anti-vGluT1 (for staining dendritic spines) and anti-MAP2 (for staining microtubules) antibodies for probing dendritic spine density showed that vGluT1-positive signals in PHNs was markedly increased after DA treatment DA, whereas ATAB administration diminished the decrease in vGluT1-positive signals (Fig. $4 \mathrm{I}$ and $\mathrm{J}$ ). These results suggested that DA impaired the production of neurotrophins and synaptogenesis through the activation of ASK1/JNK1/TNFa signaling pathway.

\subsection{DA induced inactivation of Notch pathway in neurons via TNFa}

Notch is also required for memory consolidation, a process believed to require remodeling of existing neurons in adults[35]. Several studies have shown that Notch also plays an important role in memory formation in adults, both in mammals and Drosophila[36, 37]. It is reported that Notch also plays an important role in memory formation in adults $[36,37]$. Then we addressed whether DA impacted on notch signaling via the involvement of TNFa. The expressions of NICD1/HES1 were markedly decreased in PHNs in response to higher dose of DA, whereas lower dose of DA had no significant effect on the two proteins by IB analysis (Fig. 5A and B). Parallelly, as shown by IB analysis, PHNs exposed to DA exhibited lower NICD1/HES1 levels, addition of ATAB blocked the decrease in NICD1/HES1 expression (Fig. 5C and D). ATAB PC12 cells after exposure to DA also displayed the decreased expression of NICD1/HES1, while knockdown of TNFa blocked the decrease in NICD1/HES1 expression PHNs (Fig. 5E and F). As determined by double IF staining using NICD1 antibody with MAP2 antibody, we confirmed that the decrease in NICD1 expression in PHNs in response to DA was diminished by ATAB (Fig. 5G). Immunostaining confirmed that addition of ATAB diminished the decrease in NICD1 expression in 
response to DA in PC12 cells (Fig. 5H). Parallelly, these data indicated that DA inactivated notch signaling depending on stimulation of TNFa secretion.

\subsection{DA impairs the production of neurotrophins by the inactivation of Notch pathway in vitro}

Hence, we examined whether DA had effect on the expressions of GDNF/NGF through notch signaling in vitro. As indicated by IB analysis, we also found that DA-induced the decreases in the expressions of GDNF/NGF in PHNs were significantly enhanced by addition of Notch inhibitor MK-0752 and reversed by addition of Notch activator Jagged-1 (Fig. 6A and B). Notch overexpression plasmid transfection into PC12 cells was further confirmed by western blot using the anti-Flag antibody (Fig. 6C). IB analysis revealed that the decreases in GDNF/NGF levels in PC12 cells in response to TNFa were abolished by Notch overexpression (Fig. 6D and E). Notch siRNA transfection into PC12 cells was further confirmed by western blot using the anti-Flag antibody (Fig. 6F). Furthermore, DA -mediated the decreases in Homer/synaptotagmin expression were enhanced by Notch silencing in PC12 cells (Fig. 6G and H). These data suggested that DA impaired the production of neurotrophins and synaptic formation through the inactivation of notch signaling in vitro.

\section{7 $\beta$ ASA inhibits the DA-induced the loss of neurotrophins in vitro}

BASA has been reported to have neuroprotective and synaptoprotective effects on MHE [38]. We therefore examined the effect of $\beta A S A$ on the DA-mediated downregulation of GDNF/NGF. Treatment with $\beta A S A$ abolished DA-mediated the increase in the expression of TNFa in a dose-dependent manner in PHNs (Fig. 7A and B). As determined by ELISA, DA-stimulated the release of TNFa was abated after $\beta A S A$ treatment from PHNs with dose-dependence (Fig. 7C). And the addition of $\beta A S A$ abrogated DA-induced the decrease in the notch expression with dose-dependence in PHNs (Fig. 7D and E). We then examined whether the effect of $B A S A$ on the DA-induced the loss of neurotrophins was associated with TNFa. IB analysis indicated that DA-induced the downregulation of GDNF/ synaptotagmin levels were reversed by the addition of $\beta A S A$ in PHNs, whereas, the effect of $\beta A S A$ was then inhibited by addition of TNFa or MK0752 (Fig. 7F and G). Using FM4-64 dye to probe activity-dependent synaptic vesicle recycling in PHNs revealed that synaptic activity displayed obvious reduction in response to DA, which was blocked by addition of $\beta A S A$, however, the effect of $\beta A S A$ was reversed by treatment of TNFa or MK-0752 (Fig. 7H and I). These data suggested that $\beta A S A$ diminished DA-mediated the loss of neurotrophins required for downregulation of TNFa and activation of notch signaling.

\section{8 $\beta$ ASA improves DA-induced memory impairment in MHE rats}

Then, we confirmed the effect of $\beta A S A$ on TNFa level, neurotrophic expression, and synaptic formation in vivo. It is clear from IF staining of cortices of MHE rats using TNFa combined with MAP2, pronounced increased levels in neurons in cortices of MHE rats were blocked by administration of BASA (Fig. 8A), we 
confirmed that the administration of $\beta A S A$ significantly diminished the decrease in expression of GDNF in neurons in cortices (Fig. 8B) and in expression of synaptotagmin in neurons in hippocampi (Fig. 8C) in MHE rats. Furthermore, the decreases in the NGF/synaptotagmin expressions were detected in hippocampus of MHE rats, which were amplified by DA and recovered to the normal level by administration of $\beta A S A$, furthermore, the enhancement of DA was blocked by $\beta A S A$ addition (Fig. 8D and E). Then we addressed whether $\beta A S A$ impacted on the memory decline. In YM test, SA\% in MHE rats, which were significantly lower $(P<0.01)$ than that of control rats, was enhanced by $D A$ and reversed by BASA administration, furthermore, the enhancement of DA on the SA\% of MHE rats was blocked by $\beta A S A$ addition (Fig. 8F). These data suggested that $\beta A S A$ inhibited the impairment of synaptic formation and memory function in vivo.

\section{Discussion}

Neurons regulate synaptic transmission via monitoring neuronal signaling [39], and they release inflammatory cytokines, such as TNFa [40]. TNFa is one of the major inflammatory cytokines [41] and a vital acute injury associated cytokine. Elevated baseline and systemic TNFa may have roles in lower hippocampal volume [42] and hippocampal neurodegeneration [43] that triggers cognitive changes [44]. DA directly changed the synaptic formation and elevated the expression and release of TNFa in neurons in vitro. TNFa was reported to impair memory function. Neuron with DA treatment, displayed the elevation of TNFa level in media, the impairment of synaptic formation and activity and the decrease of dendrite spine, and addition of ATAB reversed synaptic loss. MHE rats also showed the increase in the expression of TNFa, neutralization of DA blocked expression of TNFa, indicating the induction of TNFa by DA. And neutralization of TNFa diminished the synaptic formation. Furthermore, neurons exposed to DA exhibited increased production and release of TNFa levels, suggesting that neuronal TNFa in turn induced disruption of synaptogenesis. Our findings strongly suggest that the increase in DA could be responsible for enhancing synaptic loss and cognitive decline by increasing TNFa expression and secretion.

Apoptosis signal-regulating kinase 1 (ASK1) is one of the mitogen-activated protein kinase (MAPK) kinase kinase family members. c-Jun N-terminal kinase (JNK) is a member of MAPK family that is upregulated by MAPK kinase kinase family members. ASK plays an essential role in determining cell fate, including death, survival, differentiation, and mitosis [33]. The ASK1 signaling pathway is involved in the modulation of expression of pro-inflammatory cytokines through JNK1 signaling pathway $[34,45]$. Inflammatory responses are frequently mediated by the JNK signaling pathway that causes production of pro-inflammatory cytokines TNFa, and IL-6 [46]. DA which appears to be implicated in inflammatory response is its ability to induce the activation of the ASK1/JNK1 signaling pathway. Notably, evidence from DA-treated primary cultured neurons suggested that DA-induced TNFa production might be due to activation of ASK1/JNK1 pathway. Findings from TNFa-treated primary cultured neurons showed that TNFa had decreased activity of Notch signaling leading to the decreased expression of GDNF and NGF. The inhibition of ASK1/JNK1 pathway, blocked DA-induced the increase in TNFa release from neurons, inactivation of Notch pathway and impairment of synaptogenesis in neurons. 
Notch is a $320 \mathrm{kDa}$ polypeptide that is cleaved in the trans-Golgi network by a furin-like convertase to generate a functionally active receptor consisting of a $200 \mathrm{kDa}$ extracellular ligand-binding domain and a $120 \mathrm{kDa}$ transmembrane domain $[47,48]$. Activation of the Notch-1 receptor by the Jagged or Delta membrane bound ligands results in cleavage of the Notch-1 transmembrane domain to yield the Notch intracellular signaling domain (NICD). This $110 \mathrm{kDa}$ domain of Notch translocates to the nucleus and induces expression of basic helix-loop-helix transcription repressors, such as hairy/enhancer of split (Hes), in particular Hes-1 $[49,50]$. Notch is also required for memory consolidation, a process believed to require remodeling of existing neurons in adults [35]. Several studies have shown that Notch also plays an important role in memory formation in adults, both in mammals and Drosophila [36, 37]. Moreover, mice with antisense-reduced hippocampal Notch-1 mRNA and protein levels fail to sustain long-term potentiation but exhibit no defect in paired-pulse facilitation, a short-term form of synaptic plasticity [51]. Dysregulation of the Notch signaling pathway has been shown to contribute to a number of neurological diseases including Alzheimer's disease (AD) [52], cerebral autosomal dominant arteriopathy with subcortical infarcts and leukoencephalopathy (CADASIL) [53], and cortical dysplasia [54]. Our study suggests that DA induced the dysregulation of the Notch to inhibit synaptic formation through TNFa, which was responsible for the memory impairment of MHE. Counteracting of TNFa, abated DA-induced inactivation of Notch pathway and impairment of synaptogenesis in neurons. Remarkably, DA-induced impairment of synaptogenesis was reversed by activation of Notch pathway and was enhanced by the inhibition of Notch pathway in neuron. These findings suggested that DA elicited synaptic loss via ASK1/JNK1/TNFa/Notch pathway in neuron, which suggested that this cellular pathway was associated with pathogenesis of MHE.

\section{Conclusion}

In this study, we are the first demonstrating that DA levels are increased under MHE conditions and DA can exert the learning impairment. the data in vitro presents show that DA plays a role in directly triggering TNFa production and accumulation in neurons, and resulting in synaptic loss, thus reflecting that DA burden modulates dendritic spine generation and synaptic formation via TNFa release. And the signals from DA mediated via ASK1/JNK1 pathway to production of TNFa operate linked to the inactivation of Notch pathway that are sensitive to the inhibition of synaptic formation in neurons. Notably, the increase in DA was targeted to study the possible therapeutic effects of $\beta A S A$ on the associated increase in synaptic function and decrease in inflammation in MHE.

\section{Abbreviations}

AD: Alzheimer's disease; ANOVA: analysis of variance; ASK1: Apoptosis signal-regulating kinase 1; ATAB: anti-TNFa antibody; ATCC: American Type Culture Collection; A $\beta$ : amyloid $\beta$-protein; $\beta A S A$ : $\beta$-asarone; BD: Becton,Dickinson and Company; BDNF: brain-derived neurotrophic factor; $B S A$ : bovine serum albumin; CA: California; CADASIL: cerebral autosomal dominant arteriopathy with subcortical infarcts and leukoencephalopathy; CDNA: complementary Deoxyribonucleic acid; CL: contacting latency; CNS: central 
nervous system; DA: Dopamine; DL: drinking latency; DMEM: dulbecco's modified eagle medium; DNase: deoxyribonuclease; ECL: electrochemiluminescence; EL: entry latency; ELISA: enzyme-linked immunosorbent assay; FBS: fetal bovine serum ; Fig: figure; FITC: fluorescein isothiocyanate; FM4-64: N(3-triethylammom iumpropyl)-4-(p-diethylam inophenylhexatrienyl) ; GDNF囚glialcellline-derived neurotrophicfactor; HE: hepatic encephalopathy; Hes: enhancer of split; HES1: Hairy and enhancer of split1; HIV: human immunodeficiency virus; i.p. : intraperitoneally; IB: Immunoblotting; IF:

Immunofluorescence; IL-6: Interleukin- 6; JNK: c-Jun N-terminal kinase; JNK1: c-Jun N-Terminal Kinase1; MA: Massachusetts; MAP2: microtubule-associated protein 2; MAP2B: microtubule-associated protein 2B; MAPK: mitogen-activated protein kinase; MHE: Minimal hepatic encephalopathy; mRNA: Messenger Ribonucleic Acid; NGF: Nerve Growth Factor; NICD1: Notch Intracellular Domain 1; PASW: Predictive Analytics Software; PHNs: Primary hippocampal rats neurons; PVDF: Polyvinylidene fluoride; qPCR: quantitative real-time PCR; RSC96: Rat Schwann Cells; SA\%: spontaneous alternation percentage; SAMP8: Senescence-accelerated prone-8; SD: Sprague-Dawley; SD: standard deviation; SDS-PAGE: sodium dodecyl sulfate polyacrylamide gel electrophoresis; siRNA: Small interfering RNA; SPSS: Statistical Product and Service Solutions; TAA: Thioacetamide; TNFa: tumor necrosis factor-a; USA: the United States of America; VA: Virginia; vGluT1: vesicular glutamate transporter 1; WFT: water-finding task; YM: Ymaze

\section{Declarations}

\section{Acknowledgements}

We thank Dr. Haoqi Ni for optimising the image analysis method. We thank Dr. Yangping Shentu and Miss Ying Zhang for technical supports. We thank Dr. Yunchang Mo for critical reading of the manuscript.

\section{Authors' contributions}

Saidan Ding supervised the entire project and designed the research. Jian wang conceived and designed the experiments, per-formed the research interpreted, and analysed the data, and wrote the paper. Xuebao Wang conceived and designed the ex-periments, interpreted and analysed the data, and supervised all the experimental procedure. Leping Liu and He Yu performed the research and analysed the data. Xiaoai Lu, Ruimin You and Saidan Ding analysed the data and critically revised the manuscript. All authors read and approved the final manuscript.

\section{Funding}

This study was supported by the Basic Scientific Research Projects of Wenzhou (Y20180076) and Natural Science Foundation of China $(81671042,81300308)$.

\section{Availability of data and materials}


The datasets generated and/or analyzed during the current study are available from the corresponding author on reasonable request.

\section{Ethics approval}

All experiments were conducted in accordance with the guidelines for the Care and Use of Laboratory Animals of the National Institutes of Health and Institutional Animal Care and Use Committee of Wenzhou Medical University.

\section{Consent for publication}

Not applicable

\section{Competing interests}

The authors declare that they have no competing interests.

\section{References}

1. Cools, R., et al., Enhanced frontal function in Parkinson's disease. Brain : a journal of neurology, 2010. 133(Pt 1): p. 225-233.

2. Gul, A. and J. Yousaf, Effect of levodopa on frontal-subcortical and posterior cortical functioning in patients with Parkinson's disease. Singapore medical journal, 2019. 60(8): p. 414-417.

3. Ding, S., et al., Dopamine from cirrhotic liver contributes to the impaired learning and memory ability of hippocampus in minimal hepatic encephalopathy. Hepatol Int, 2013. 7(3): p. 923-36.

4. Ding, S., et al., The inactivation of JAK2/STAT3 signaling and desensitization of M1 mAChR in minimal hepatic encephalopathy (MHE) and the protection of naringin against MHE. Cell Physiol Biochem, 2014. 34(6): p. 1933-50.

5. Ding, S., et al., Elevated intracranial dopamine impairs the glutamate?nitric oxide?cyclic guanosine monophosphate pathway in cortical astrocytes in rats with minimal hepatic encephalopathy. 2014. 10(3): p. 1215--1224.

6. Saidan, et al., Baicalin reverses the impairment of synaptogenesis induced by dopamine burden via the stimulation of GABA A R-TrkB interaction in minimal hepatic encephalopathy. 2018.

7. Ding, S., et al., Dopamine induces glutamate accumulation in astrocytes to disrupt neuronal function leading to pathogenesis of minimal hepatic encephalopathy. 2017: p. S0306452217306930.

8. Ding, S., et al., Dopamine Burden Induced the Inactivation of Sonic Hedgehog Signaling to Cognitive Decline in Minimal Hepatic Encephalopathy. 2017. 8(4).

9. Ding, S., et al., Dopamine Burden Triggers Neurodegeneration via Production and Release of TNFalpha from Astrocytes in Minimal Hepatic Encephalopathy. Mol Neurobiol, 2016. 53(8): p. 5324-43.

10. JE, M., Effects of interleukin-1 and tumor necrosis factor-alpha on astrocytes, microglia, oligodendrocytes, and glial precursors in vitro. Developmental neuroscience, 1991. 13(3): p. 130-7. 
11. EC, B., et al., Control of synaptic strength by glial TNFalpha. Science (New York, N.Y.), 2002. 295(5563): p. 2282-5.

12. MK, M. and T. MG, TNF signaling inhibition in the CNS: implications for normal brain function and neurodegenerative disease. Journal of neuroinflammation, 2008. 5(undefined): p. 45.

13. R, P., et al., Proinflammatory cytokines in sera of elderly patients with dementia: levels in vascular injury are higher than those of mild-moderate Alzheimer's disease patients. Experimental gerontology, 2002. 37(null): p. 257-63.

14. M, M., et al., Tumor necrosis factor-alpha (TNF-alpha) increases both in the brain and in the cerebrospinal fluid from parkinsonian patients. Neuroscience letters, 1994. 165(null): p. 208-10.

15. SL, W., et al., Intracerebral cytokine messenger RNA expression in acquired immunodeficiency syndrome dementia. Annals of neurology, 1993. 33(6): p. 576-82.

16. Casadesus, G., et al., Increased isoprostane and prostaglandin are prominent in neurons in Alzheimer disease. Molecular Neurodegeneration, 2007. 2(1): p. 2.

17. Marquette, C., et al., Brain interleukin-1 receptors during rabies virus infection. Journal of Neuroimmunology, 1994. 54(1-2): p. 179-179.

18. Montine, T.J., et al., Elevated CSF prostaglandin E2 levels in patients with probable AD. Neurology, 2000. 55(2): p. 1495.

19. Xu, F., et al., Pro-neurogenic effect of $\beta$-asarone on RSC96 Schwann cells in vitro. In vitro cellular \& developmental biology. Animal, 2016. 52(3): p. 278-286.

20. Geng, Y., et al., Beta-asarone improves cognitive function by suppressing neuronal apoptosis in the beta-amyloid hippocampus injection rats. Biol Pharm Bull, 2010. 33(5): p. 836-43.

21. Chen, Y., et al., $\beta$-Asarone prevents autophagy and synaptic loss by reducing ROCK expression in asenescence-accelerated prone 8 mice. Brain Res, 2014. 1552: p. 41-54.

22. Wei, G., et al., $\beta$-Asarone inhibits neuronal apoptosis via the CaMKII/CREB/BCl-2 signaling pathway in an in vitro model and ABPP/PS1 mice. J Alzheimers Dis, 2013. 33(3): p. 863-80.

23. Liu, S.J., et al., Neuroprotective effect of $\beta$-asarone against Alzheimer's disease: regulation of synaptic plasticity by increased expression of SYP and GluR1. Drug Des Devel Ther, 2016. 10: $\mathrm{p}$. 1461-9.

24. Lee, B., et al., Effect of Beta-Asarone on Impairment of Spatial Working Memory and Apoptosis in the Hippocampus of Rats Exposed to Chronic Corticosterone Administration. Biomol Ther (Seoul), 2015. 23(6): p. 571-81.

25. Yang, Q.Q., et al., $\beta$-Asarone Rescues Pb-Induced Impairments of Spatial Memory and Synaptogenesis in Rats. PLoS One, 2016. 11(12): p. e0167401.

26. Albrecht, J., et al., Extracellular concentrations of taurine, glutamate, and aspartate in the cerebral cortex of rats at the asymptomatic stage of thioacetamide-induced hepatic failure: modulation by ketamine anesthesia. Neurochem Res, 2000. 25(11): p. 1497-502. 
27. Yamada, M., et al., Implanted cannula-mediated repetitive administration of Abeta25-35 into the mouse cerebral ventricle effectively impairs spatial working memory. Behav. Brain Res., 2005. 164(2): p. 139-46.

28. Kawasumi, M., et al., Targeted introduction of V642I mutation in amyloid precursor protein gene causes functional abnormality resembling early stage of Alzheimer's disease in aged mice. Eur. J. Neurosci., 2004. 19(10): p. 2826-2838.

29. Satomura, Y., et al., Measurement of serum PSP/reg-protein concentration in various diseases with a newly developed enzyme-linked immunosorbent assay. Journal of gastroenterology, 1995. 30(5): p. 643-650.

30. Graf, R., et al., Coordinate regulation of secretory stress proteins (PSP/reg, PAP I, PAP II, and PAP III) in the rat exocrine pancreas during experimental acute pancreatitis. Journal of Surgical Research, 2002. 105(2): p. 136-144.

31. Lawrance, I.C., C. Fiocchi, and S. Chakravarti, Ulcerative colitis and Crohn's disease: distinctive gene expression profiles and novel susceptibility candidate genes. Human Molecular Genetics, 2001. 10(5): p. 445-456.

32. Dieckgraefe, B., et al., Analysis of mucosal gene expression in inflammatory bowel disease by parallel oligonucleotide arrays. Physiological genomics, 2000. 4(1): p. 1-11.

33. Nagai, H., et al., Pathophysiological roles of ASK1-MAP kinase signaling pathways. BMB Reports, 2007. 40(1): p. 1-6.

34. Davis, R.J., Signal transduction by the JNK group of MAP kinases. Cell, 2000. 103(2): p. 239-252.

35. Bailey, C.H., D. Bartsch, and E.R. Kandel, Toward a molecular definition of long-term memory storage. Proceedings of the National Academy of Sciences, 1996. 93(24): p. 13445-13452.

36. Costa, R.M., T. Honjo, and A.J. Silva, Learning and memory deficits in Notch mutant mice. Current biology, 2003. 13(15): p. 1348-1354.

37. Presente, A., et al., Notch is required for long-term memory in Drosophila. Proceedings of the National Academy of Sciences of the United States of America, 2004. 101(6): p. 1764-1768.

38. Ding, S., et al., Baicalin reverses the impairment of synaptogenesis induced by dopamine burden via the stimulation of GABA A R-TrkB interaction in minimal hepatic encephalopathy. Psychopharmacology, 2018. 235(4): p. 1163-1178.

39. Abbott, N.J., L. Ronnback, and E. Hansson, Astrocyte-endothelial interactions at the blood-brain barrier. Nat. Rev. Neurosci., 2006. 7(1): p. 41-53.

40. Qin, H. and E.N. Benveniste, ELISA methodology to quantify astrocyte production of cytokines/chemokines in vitro. Methods Mol. Biol., 2012. 814: p. 235-49.

41. Y, S., et al., Distinct destructive signal pathways of neuronal death in Alzheimer's disease. Trends in molecular medicine, 2006. 12(12): p. 574-9.

42. KD, S., et al., Cortisol, cytokines, and hippocampal volume interactions in the elderly. Frontiers in aging neuroscience, 2014. 6(undefined): p. 153. 
43. Montgomery, S.L., et al., Ablation of TNF-RI/RII expression in Alzheimer's disease mice leads to an unexpected enhancement of pathology: implications for chronic pan-TNF-a suppressive therapeutic strategies in the brain. American Journal of Pathology, 2011. 179(4): p. 2053-2070.

44. N, T., et al., Tumor necrosis factor-alpha triggers a cytokine cascade yielding postoperative cognitive decline. Proceedings of the National Academy of Sciences of the United States of America, 2010. 107(47): p. 20518-22.

45. Gupta, S., et al., Selective interaction of JNK protein kinase isoforms with transcription factors. The EMBO journal, 1996. 15(11): p. 2760.

46. Han, M.S., et al., JNK expression by macrophages promotes obesity-induced insulin resistance and inflammation. Science, 2013. 339(6116): p. 218-222.

47. Blaumueller, C.M., et al., Intracellular cleavage of Notch leads to a heterodimeric receptor on the plasma membrane. Cell, 1997. 90(2): p. 281-291.

48. Logeat, F., et al., The Notch1 receptor is cleaved constitutively by a furin-like convertase. Proceedings of the National Academy of Sciences, 1998. 95(14): p. 8108-8112.

49. Jarriault, S., et al., Signalling downstream of activated mammalian Notch. Nature, 1995. 377(6547): p. 355.

50. Ohtsuka, T., et al., Hes 1 and Hes5 as notch effectors in mammalian neuronal differentiation. The EMBO journal, 1999. 18(8): p. 2196-2207.

51. Wang, Y., et al., Involvement of Notch signaling in hippocampal synaptic plasticity. Proceedings of the National Academy of Sciences of the United States of America, 2004. 101(25): p. 9458-9462.

52. Rui, M.C., et al., Learning and Memory Deficits in Notch Mutant Mice. Current Biology Cb, 2003. 13(15): p. 1348-54.

53. Abe, K., et al., Clinical Features of CADASIL. Annals of the New York Academy of Sciences, 2002. 977(1): p. 266.

54. Hartmann, D., S.B. De, and P. Saftig, Presenilin-1 deficiency leads to loss of Cajal-Retzius neurons andcortical dysplasia similar to human type 2 lissencephaly. Current Biology Cb, 1999. 9(14): p. 719727.

\section{Figures}



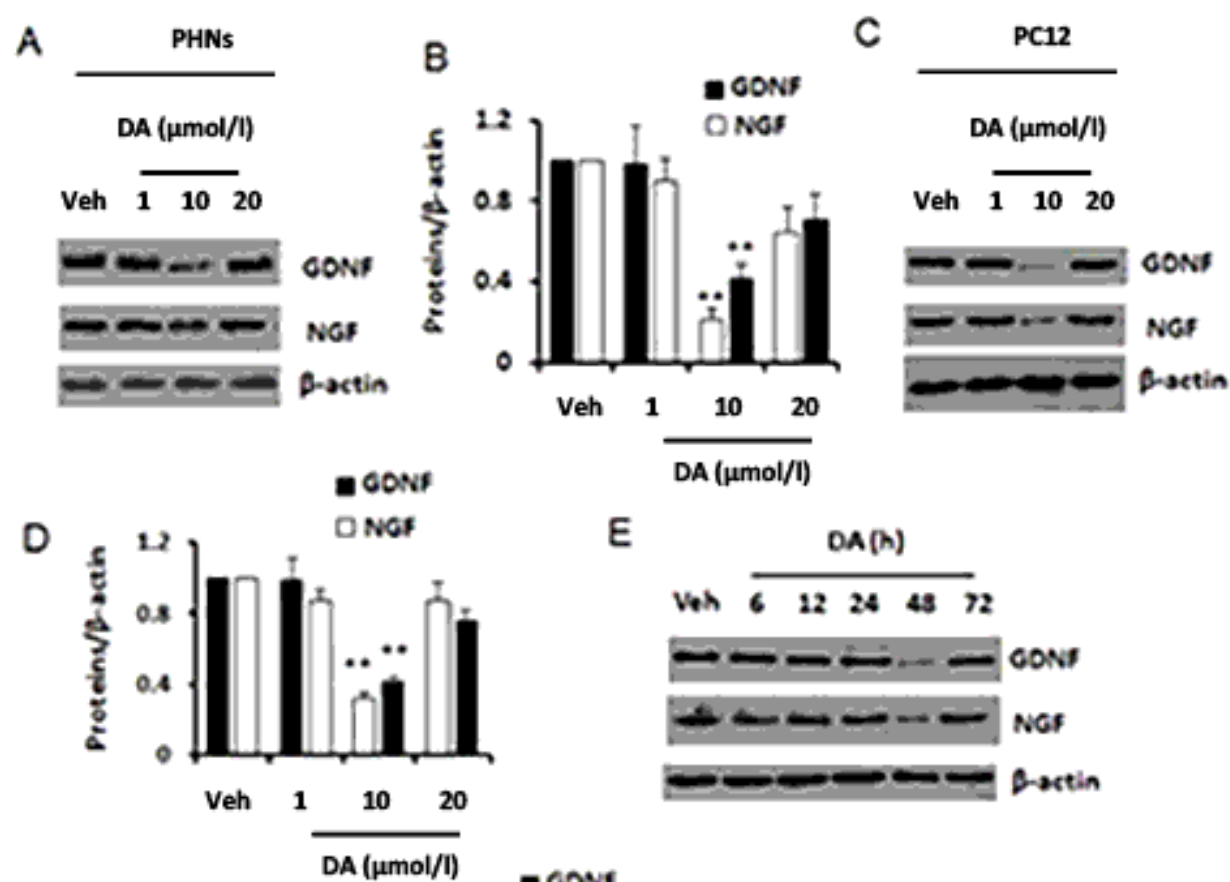

E
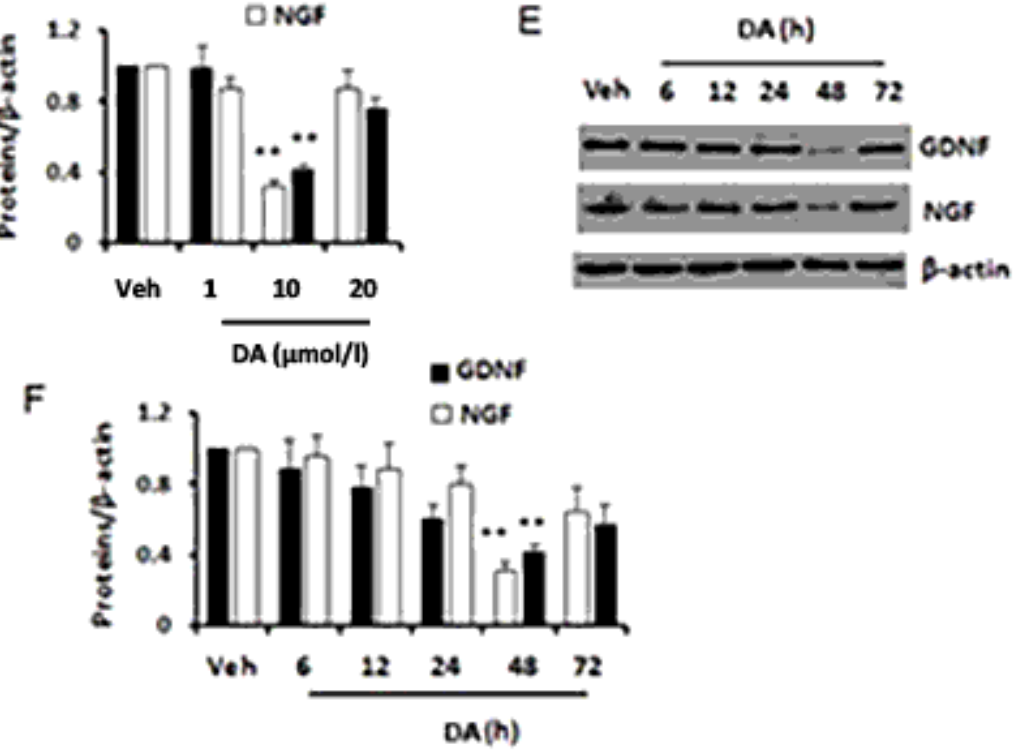

G

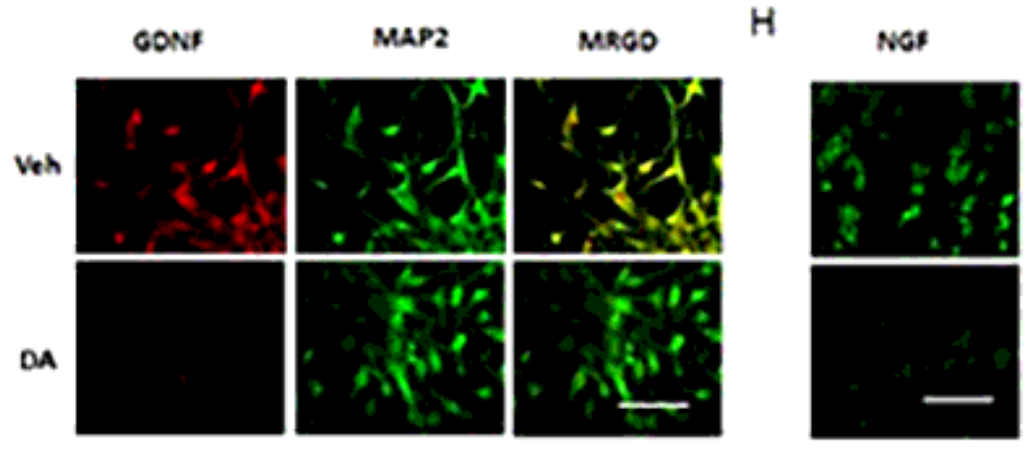

Figure 1

DA impairs synaptic formation in vitro. (A-D) IB analysis and subsequent densitometry of lysate from PHNs $(A, B)$ and PC12 cells. (C,D) stimulated with various concentrations of DA using anti-GDNF/NGF and anti- $\beta$-actin antibodies. (E,F) IB analysis of lysate from PHNs stimulated with $2.5 \mathrm{mmol} / \mathrm{L}$ DA for various time points using anti-GDNF/NGF and anti- $\beta$-actin antibodies and subsequent densitometry. (G) Immunostaining of PHNs stimulated with DA using antibodies against GDNF (red), MAP2 (green). (H) Immunostaining of PC12 cells stimulated with DA using antibodies against NGF. Data are shown as mean $\pm S D$. ${ }^{*} P<0.05,{ }^{*} P<0.01$ vs vehicle-treated group. veh, vehicle. Scale bar, $25 \mu \mathrm{m}$. MRGD, merged image. 
A

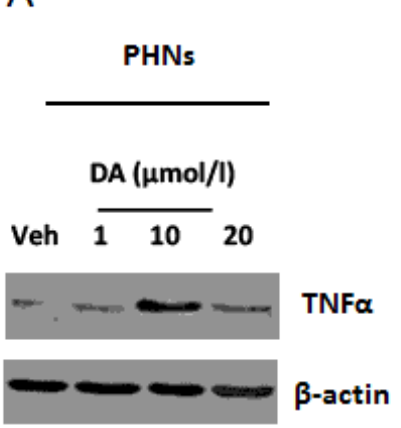

B

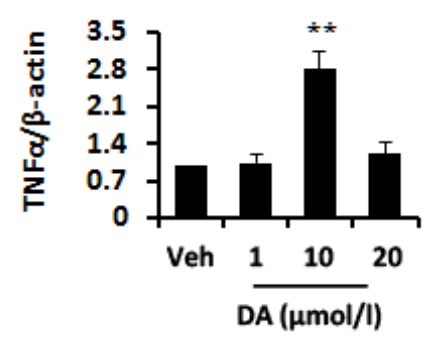

C

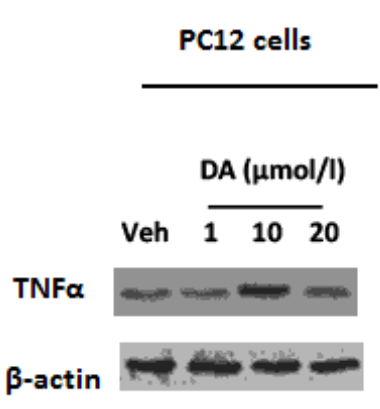

E

F
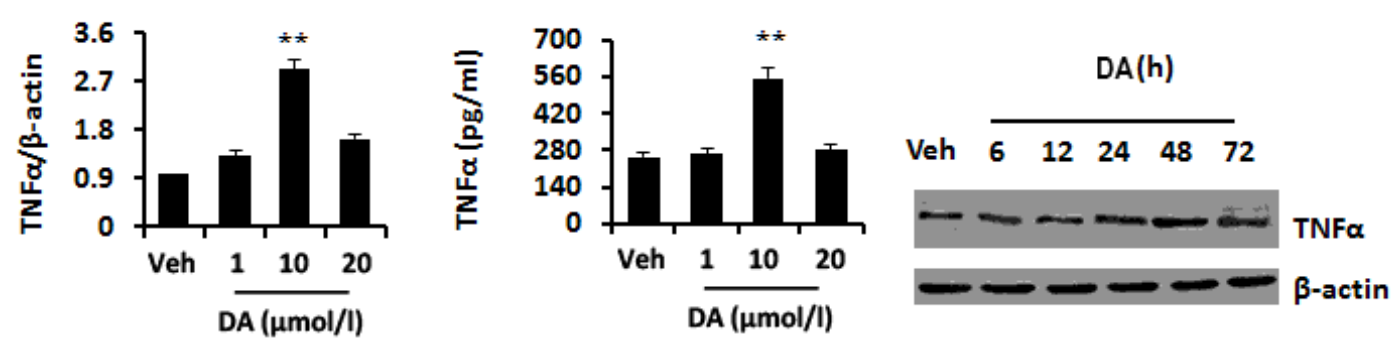

G

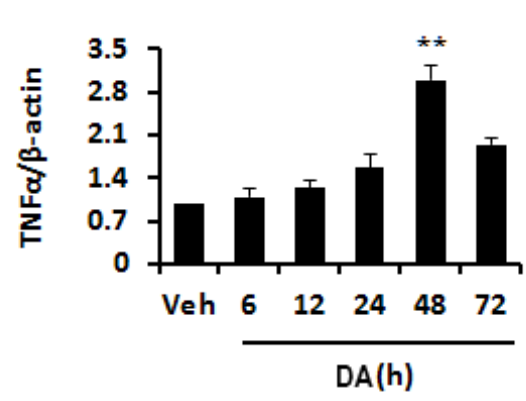

I

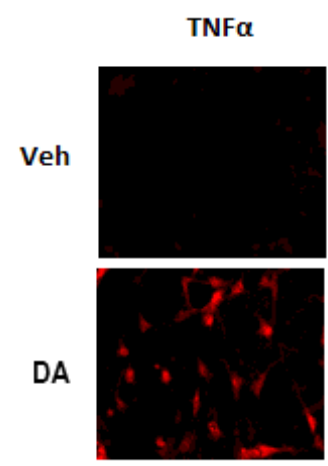

MAP2
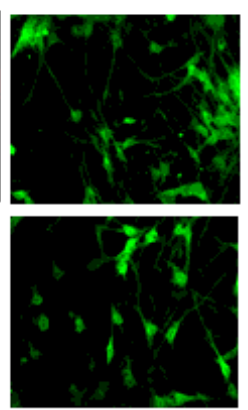

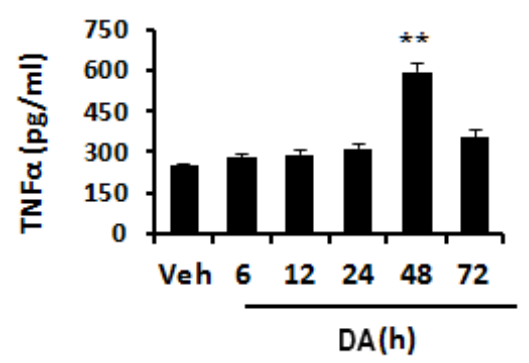

J

MRGD

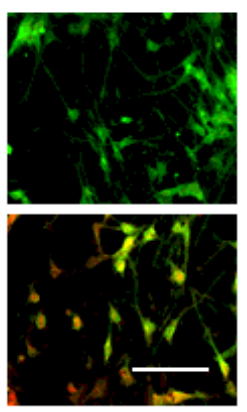

TNF $\alpha$

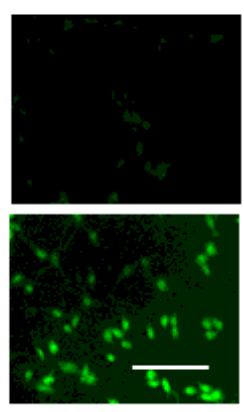

\section{Figure 2}

DA elicits the production of TNFa in neurons in vitro. (A-D) IB analysis of lysate from PHNs $(A, B)$ and PC12 cells (C,D) stimulated with various concentrations of DA using anti-TNFa and anti- $\beta$-actin antibodies and subsequent densitometry. (E) ELISA assay for TNFa level of Supernatants from PHNs stimulated with various concentrations of $D A$. $(F, G)$ IB analysis of lysate from PHNs stimulated with 2.5 mmol/L DA for various time points using anti- TNFa and anti- $\beta$-actin antibodies and subsequent 
densitometry. (H) ELISA assay for TNFa level of Supernatants from PHNs stimulated with $2.5 \mathrm{mmol} / \mathrm{L}$ DA for various time points. (I) Immunostaining of PHNs stimulated with DA using antibodies against TNFa (red), MAP2 (green). (J) Immunostaining of PC12 cells stimulated with DA using antibodies against TNFa. Data are shown as mean \pm SD. ${ }^{*} P<0.05,{ }^{*} \mathrm{P}<0.01$ vs vehicle-treated group. veh, vehicle. Scale bar, $25 \mu \mathrm{m}$. MRGD, merged image.

A

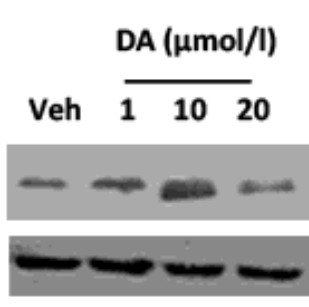

PASK1

ASK1
B

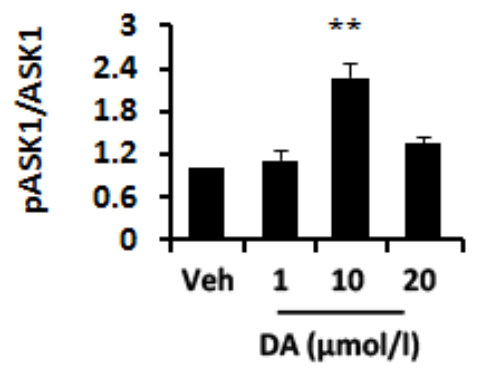

$\mathrm{F}$

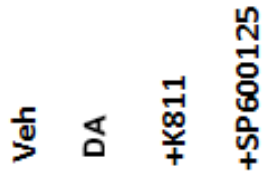

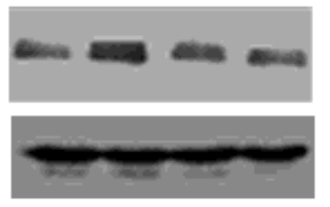

TNF $\alpha$

$\beta$-actin

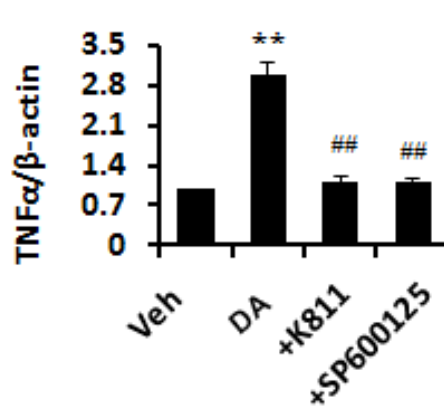

C

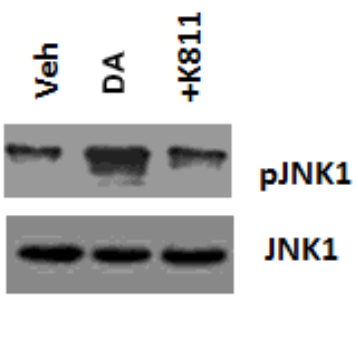

G

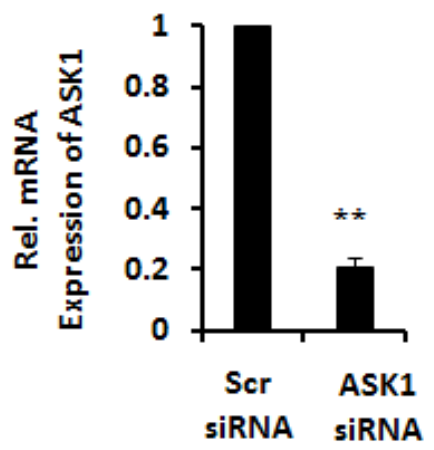

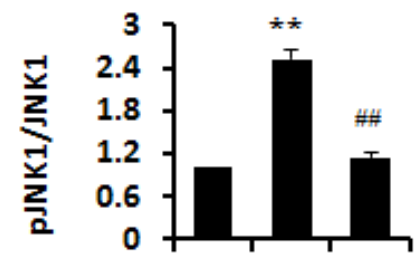

ver $0^{p}+0^{8 t^{x}}$

$\mathrm{H}$

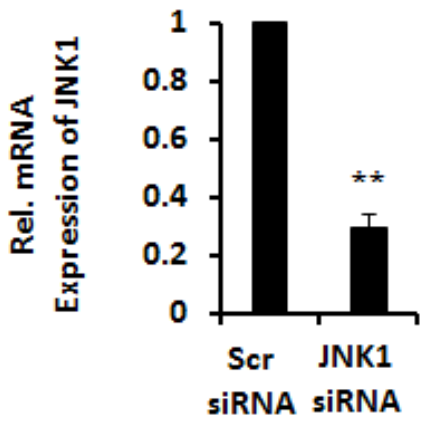

$\mathrm{K}$

Scr SIRNA ASK siRNA JNK siRNA

Veh DA Veh DA Veh DA

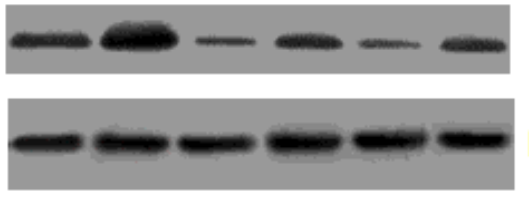

TNF $\alpha$

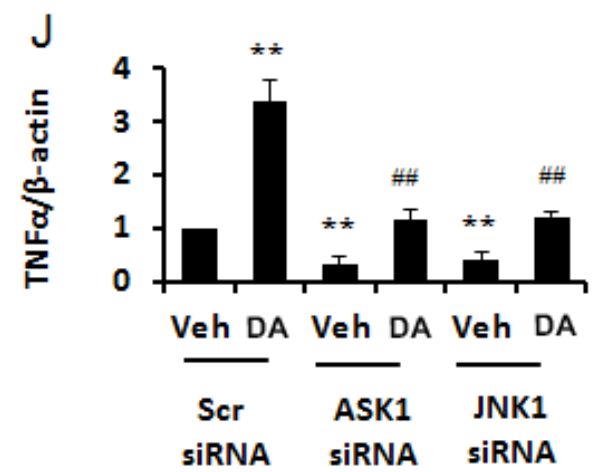

$\beta$-actin

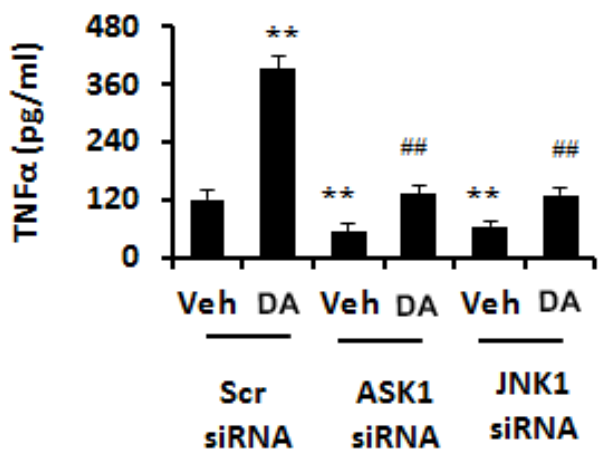

Figure 3

DA triggers TNFa production via activation of ASK1/JNK1 signaling in neurons. $(A, B)$ IB analysis of PHNs stimulated with various concentrations of DA $(10,50,100 \mathrm{nmol} / \mathrm{I})$ using anti- pASK1 and anti-ASK1 antibodies and subsequent densitometry. (C,D) IB analysis of PHNs stimulated with 100nmol/I DA alone or after preincubation with K811 using antibodies against pJNK1 and JNK1 and subsequent densitometry. (E,F) IB analysis of lysate from PHNs stimulated with $100 \mathrm{nmol} / \mathrm{I}$ DA in the presence of K811 or SP600125 using anti-TNFa and anti- $\beta$-actin antibodies and subsequent densitometry. (G,H) RT- 
PCR analysis of ASK1(G)/JNK1(H) mRNAs of PC12 cells transfected with ASK1 siRNA or JNK1 siRNA. $(\mathrm{I}, \mathrm{J})$ IB analysis of lysate from PC12 cells stimulated with $100 \mathrm{nmol} / \mathrm{I}$ DA after transfection with ASK1 siRNA or JNK1 siRNA using anti-TNFa and anti- $\beta$-actin antibodies and subsequent densitometry. (K) ELISA assay for TNFa level of Supernatants from PC12 cells stimulated with $100 \mathrm{nmol} / \mathrm{I}$ DA after transfection with ASK1 siRNA or JNK1 siRNA. Data are shown as mean \pm SD. ${ }^{*} P<0.05,{ }^{\star *} P<0.01$ vs vehicle-treated group. \#P $<0.05$, \#\#P $<0.01$ vs DA-treated group. Scr, scrambled. veh, vehicle. n.s., not significant vs vehicle-treated group. Scale bar, $25 \mu \mathrm{m}$. MRGD, merged image.

A

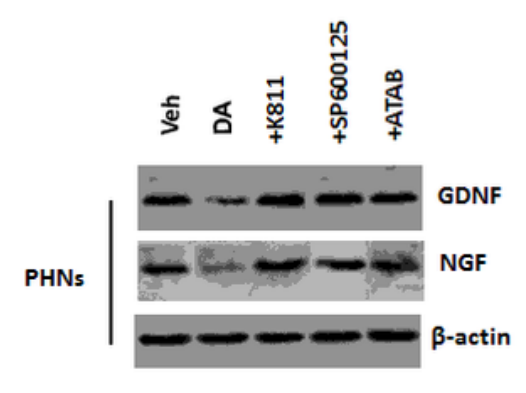

D

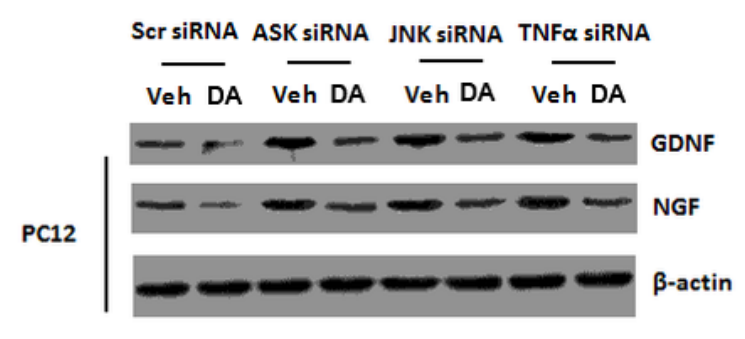

B

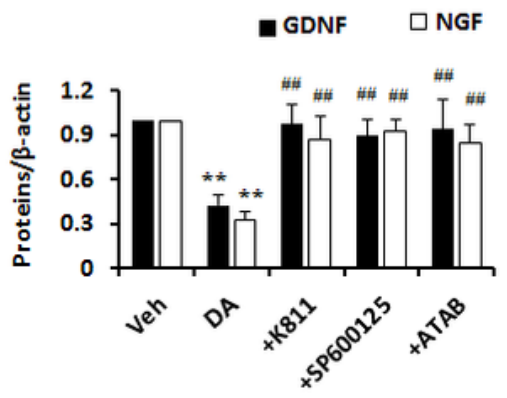

C

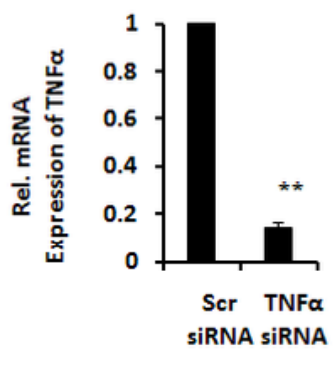

E

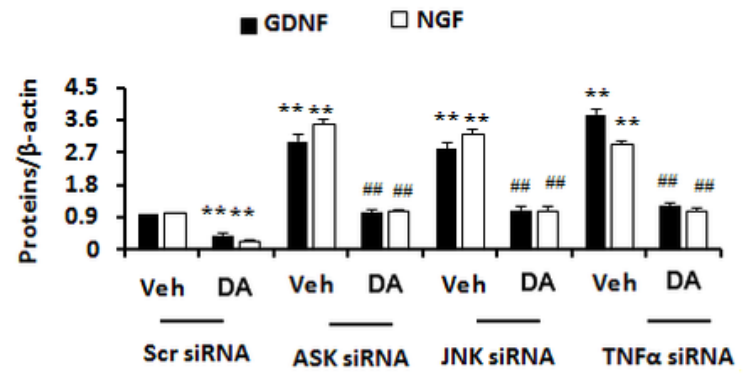

G

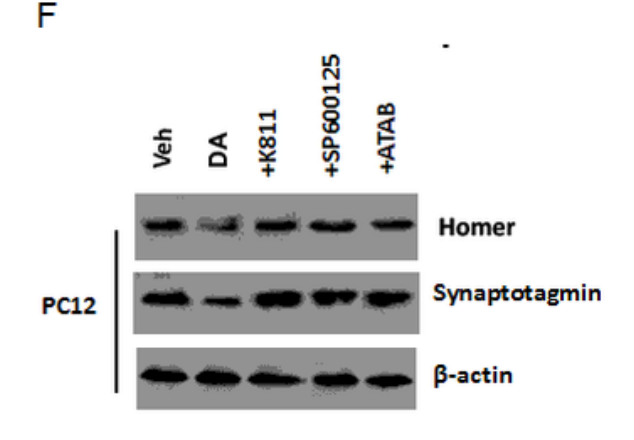

$\mathrm{H}$

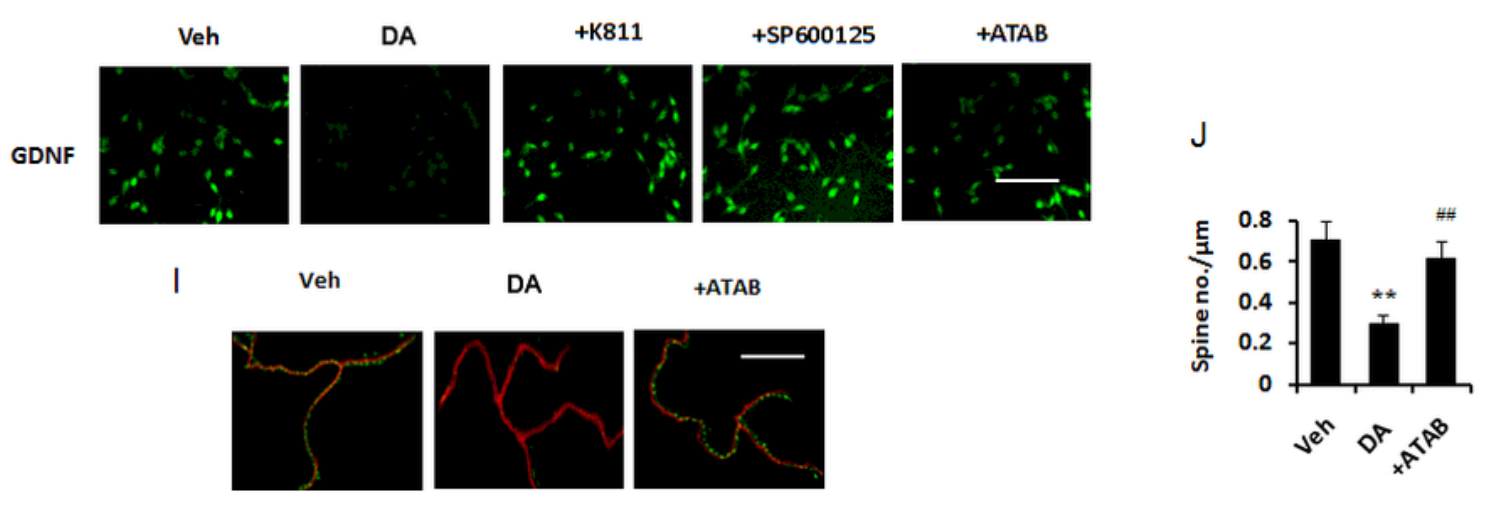




\section{Figure 4}

DA induces synaptic loss via upregulation of TNFa in vitro. (A,B) IB analysis of lysates from PHNs stimulated with DA after preincubation with K811, SP600125 or ATAB using anti- GDNF/NGF and anti- $\beta$ actin antibodies and subsequent densitometry. (C) RT-PCR analysis of TNFa mRNAs of PC12 cells transfected with TNFa siRNA. (D,E) IB analysis of lysates from PC12 cells stimulated with $100 \mathrm{nmol} / \mathrm{I}$ DA after transfection with ASK1/JNK1/TNFa siRNA using anti- GDNF/NGF and anti- $\beta$-actin antibodies and subsequent densitometry. $(F, G)$ IB analysis of lysates from PHNs stimulated wit DA after preincubation with K811, SP600125 or ATAB using anti-Homer/synaptotagmin and anti- $\beta$-actin antibodies and subsequent densitometry. $(H)$ Immunostaining of PC12 cells stimulated with $100 \mathrm{nmol} / \mathrm{I} D A$ in the presence of K811, SP600125 or ATAB using antibodies against GDNF. $(I, J)$ Immunostaining of PHNs stimulated with 100nmol/I DA after preincubation with ATAB against MAP2B (red), vGluT1 (green). Red signals indicate MAP2B for microtubule staining and green signals indicate vGluT1 for detecting excitatory synapses. Synaptic density was analyzed by counting green signals (vGluT1-positive dendritic spines) using Image $J$, and expressed per $1 \mu \mathrm{m}$ of apical dendrite. Data are shown as mean $\pm S D$. ${ }^{*} P<0.05$, $\star \star P<0.01$ vs vehicle-treated group. \#P $<0.05$, \#\#P $<0.01$ vs DA-treated group. Scr, scrambled. veh, vehicle. Scale bar, $25 \mu \mathrm{m}$. MRGD, merged image. 
A

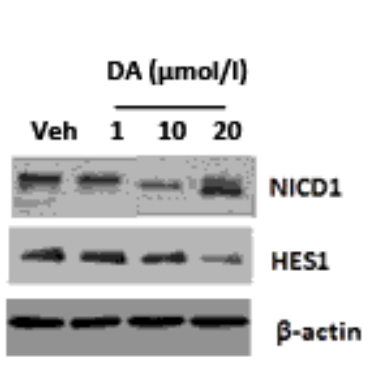

B

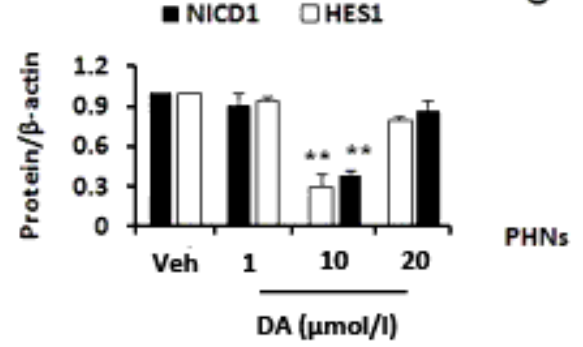

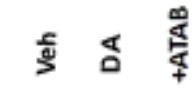

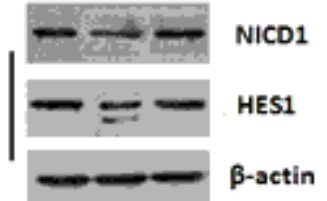

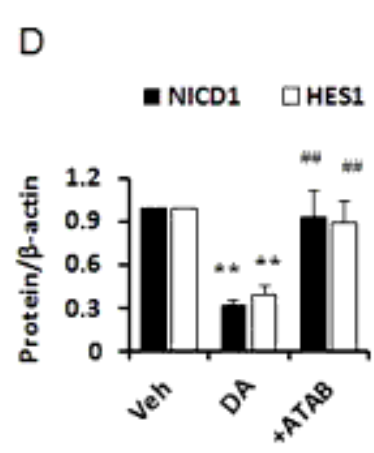

G

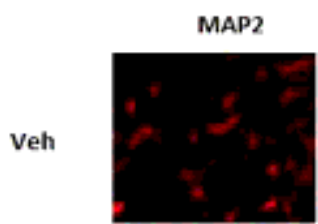

DA
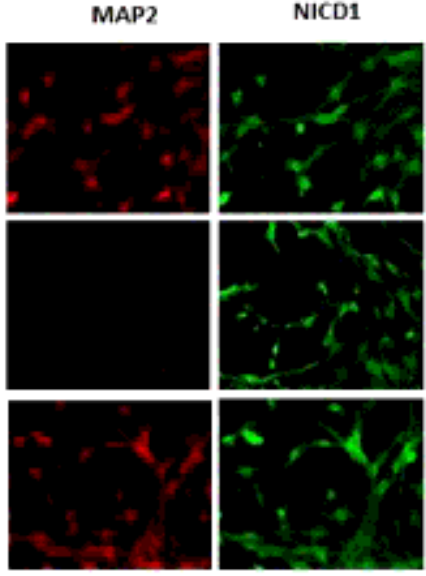

$\mathrm{E}$

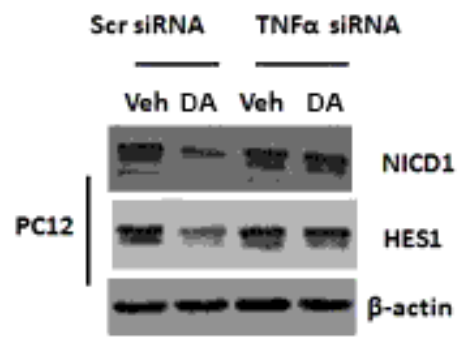

$\mathrm{F}$

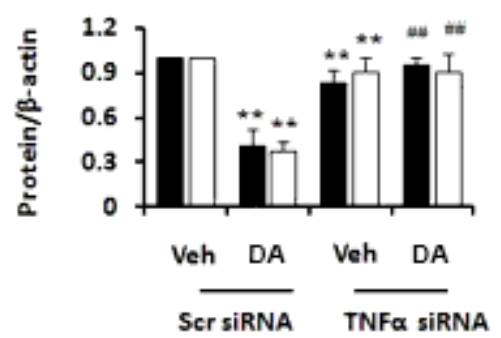

n NICD1 DHES1

$\mathrm{H}$

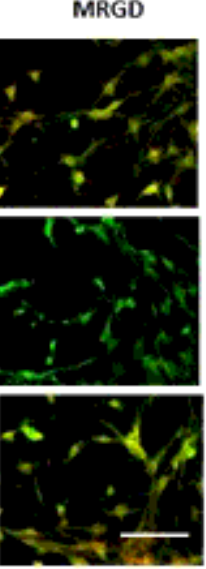

NICD1

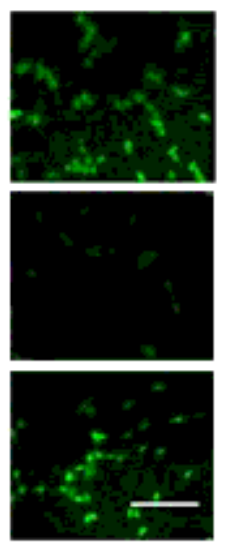

\section{Figure 5}

DA inactivates notch signaling pathway via upregulation of TNFa $(A, B)$ IB analysis of lysate from PHNs stimulated with various concentrations of DA using anti-NICD1/HES1 and anti- $\beta$-actin antibodies and subsequent densitometry. (C,D) IB analysis of lysates from PHNs stimulated with $100 \mathrm{nmol} / \mathrm{I}$ DA after preincubation with ATAB using anti- NICD1/HES1 and anti- $\beta$-actin antibodies and subsequent densitometry. (E,F) IB analysis of lysates from PC12 cells stimulated with 100nmol/I DA after transfection with TNFa siRNA using anti- NICD1/ HES1 and anti- $\beta$-actin antibodies and subsequent densitometry. (G) Immunostaining of PHNs stimulated with 100nmol/I DA in the presence of ATAB using antibodies against NICD1 (red), MAP2 (green). (H) Immunostaining of PC12 cells stimulated with $100 \mathrm{nmol} / \mathrm{I} D A$ in the presence of ATAB using antibodies against NICD1. Data are shown as mean \pm SD. *P $<0.05$, ${ }^{\star *} \mathrm{P}<0.01$ vs vehicle-treated group. \#P $<0.05$, \#\#P $<0.01$ vs $D A$-treated group. Scr, scrambled. veh, vehicle. Scale bar, $25 \mu \mathrm{m}$. MRGD, merged image. 
A
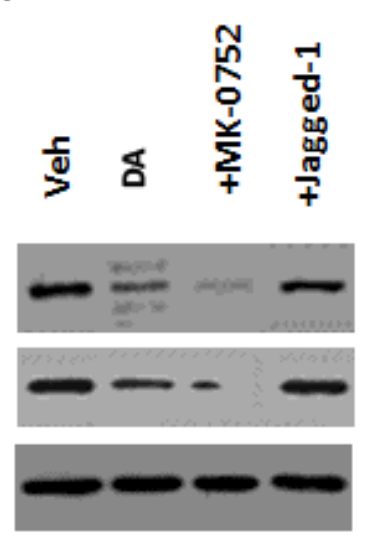

GDNF

NGF

$\beta$-actin

D

Flag Flag-Notch1

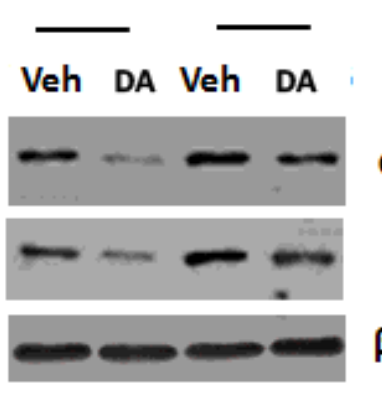

G

Scr siRNA Notch1 siRNA

E

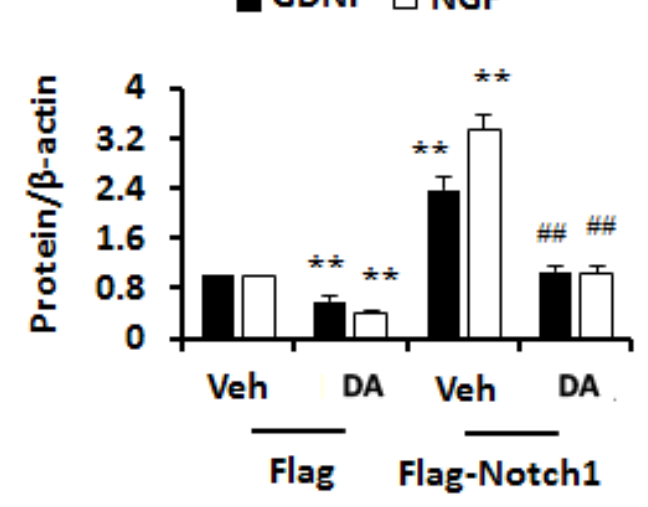

$\mathrm{H}$
C

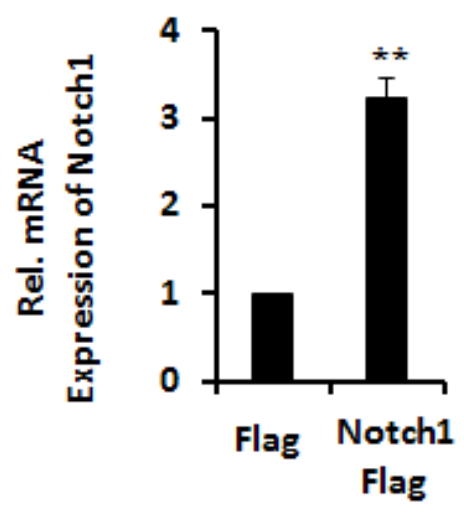

F

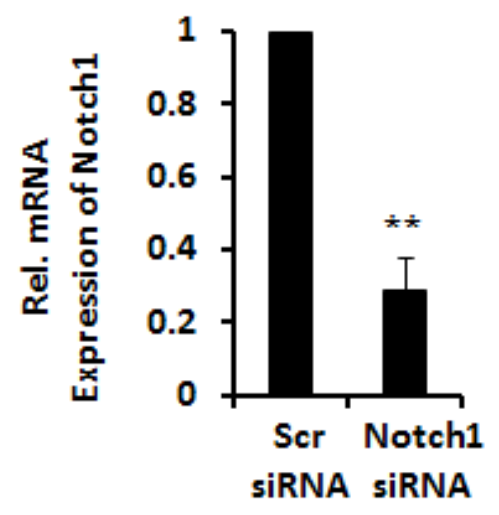

Veh DA Veh DA

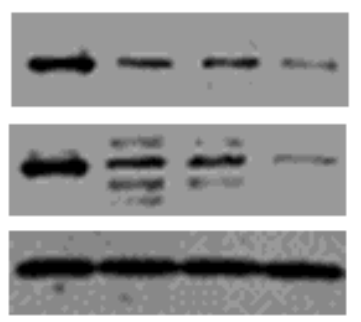

Homer
Synaptotagmin

$\beta$-actin

Homer

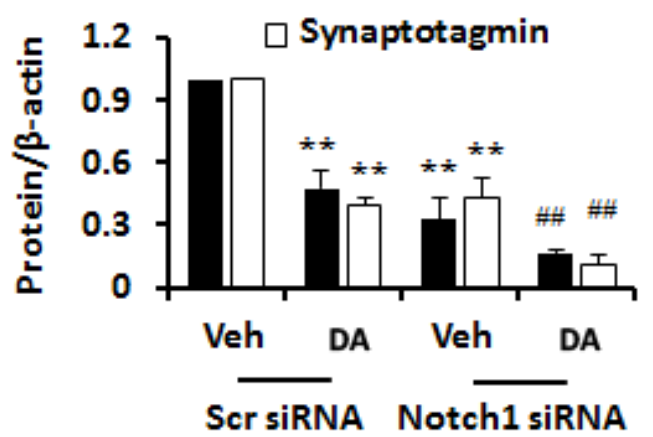

Figure 6

DA induces synaptic loss via inactivation of Notch signaling pathway in vitro. $(A, B)$ IB analysis of lysate from PHNs stimulated with 100nmol/I DA in the presence of MK-0752 or Jagged-1 using anti-GDNF/NGF and anti- $\beta$-actin antibodies and subsequent densitometry. (C) RT-PCR analysis of notch mRNA of PC12 cells transfected with notch overexpression plasmid. (D,E) IB analysis of lysate from PC12 cells stimulated with 100nmol/I DA after transfection with Notch1 overexpression plasmid using anti- 
GDNF/NGF and anti- $\beta$-actin antibodies and subsequent densitometry. (F) RT-PCR analysis of notch mRNA of PC12 cells transfected with notch siRNA. $(\mathrm{G}, \mathrm{H})$ IB analysis of lysate from PC12 cells stimulated with 100nmol/I DA after transfection with Notch1 siRNA using anti- Homer/synaptotagmin and anti- $\beta$-actin antibodies and subsequent densitometry. Data are shown as mean \pm SD. $* P<0.05, * * P<0.01$ vs vehicletreated group. \#P<0.05, \#\#P<0.01 vs TNFa-treated group. Scr, scrambled. veh, vehicle. MRGD, merged image.

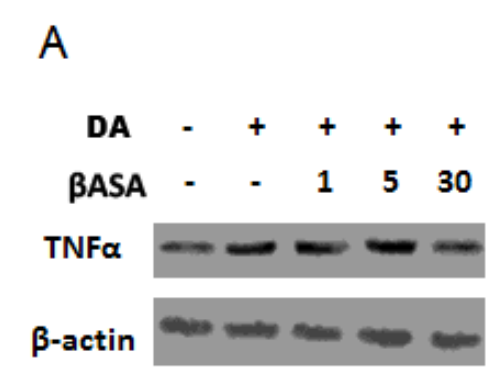

D

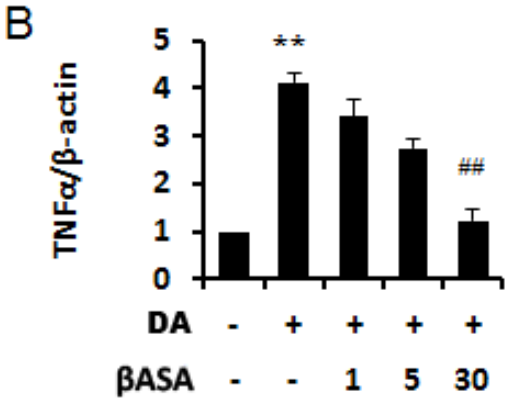

E
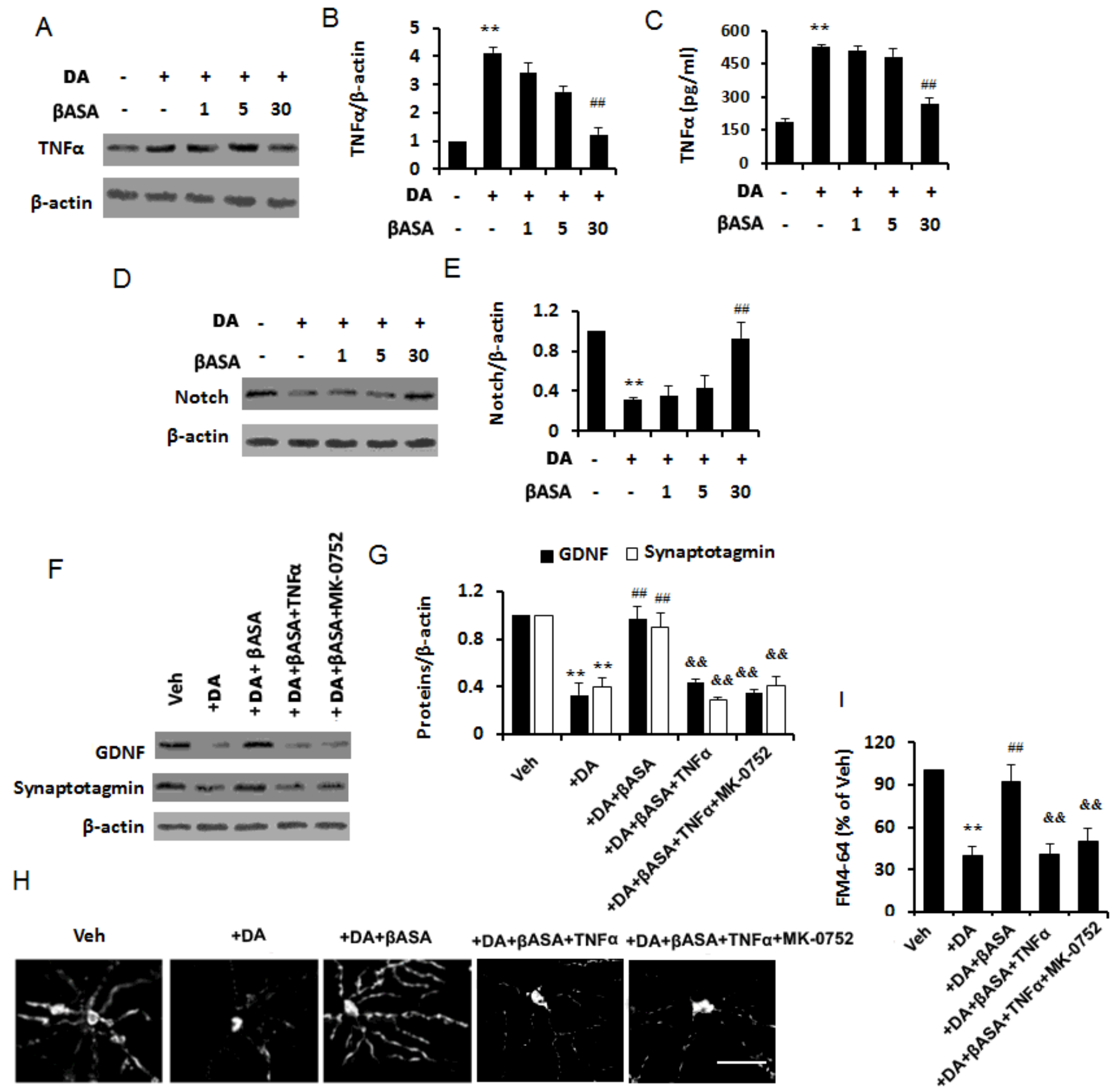

Figure 7 
BASA attenuates DA-induced synaptic loss via downregulation of TNFa (A.B) IB analysis of lysate from PHNs stimulated with $100 \mathrm{nmol} / \mathrm{I}$ DA in the presence of various concentration of $\beta A S A$ using anti- TNFa and anti- $\beta$-actin antibodies and subsequent densitometry. (C) ELISA assay for TNFa level of Supernatants from PHNs stimulated with $100 \mathrm{nmol} / \mathrm{I} D A$ in the presence of $\beta A S A$. (D,E) IB analysis of lysate from PHNs stimulated with $100 \mathrm{nmol} / \mathrm{I}$ DA in the presence of various concentration of $\beta A S A$ using anti- Notch and anti- $\beta$-actin antibodies and subsequent densitometry. $(F, G)$ IB analysis of lysate from PHNs stimulated with $100 \mathrm{nmol} / \mathrm{I}$ DA in the presence of $\beta$ ASA together with TNFa or MK-0752 using antiGDNF/synaptotagmin and anti- $\beta$-actin antibodies and subsequent densitometry. $(H, I)$ Representative image of FM4-64 staining of functional presynaptic terminals in PHNs stimulated with 100 $\mathrm{nmol} / \mathrm{I}$ DA in the presence of $\beta A S A$ together with TNFa or MK-0752. The panel $\mathrm{H}$ indicates that quantitative analysis of changes on average in FM4-64 puncta intensity. Data are shown as mean $\pm S D$. ${ }^{*} P<0.05,{ }^{\star} \mathrm{P}<0.01 \mathrm{vs}$ vehicle-treated group. \#P $<0.05$, \#\#P $<0.01$ vs $D A$-treated group, \&P $<0.05, \& \& P<0.01$ vs $D A+\beta A S A$-treated group. Scr, scrambled. veh, vehicle. MRGD, merged image. 

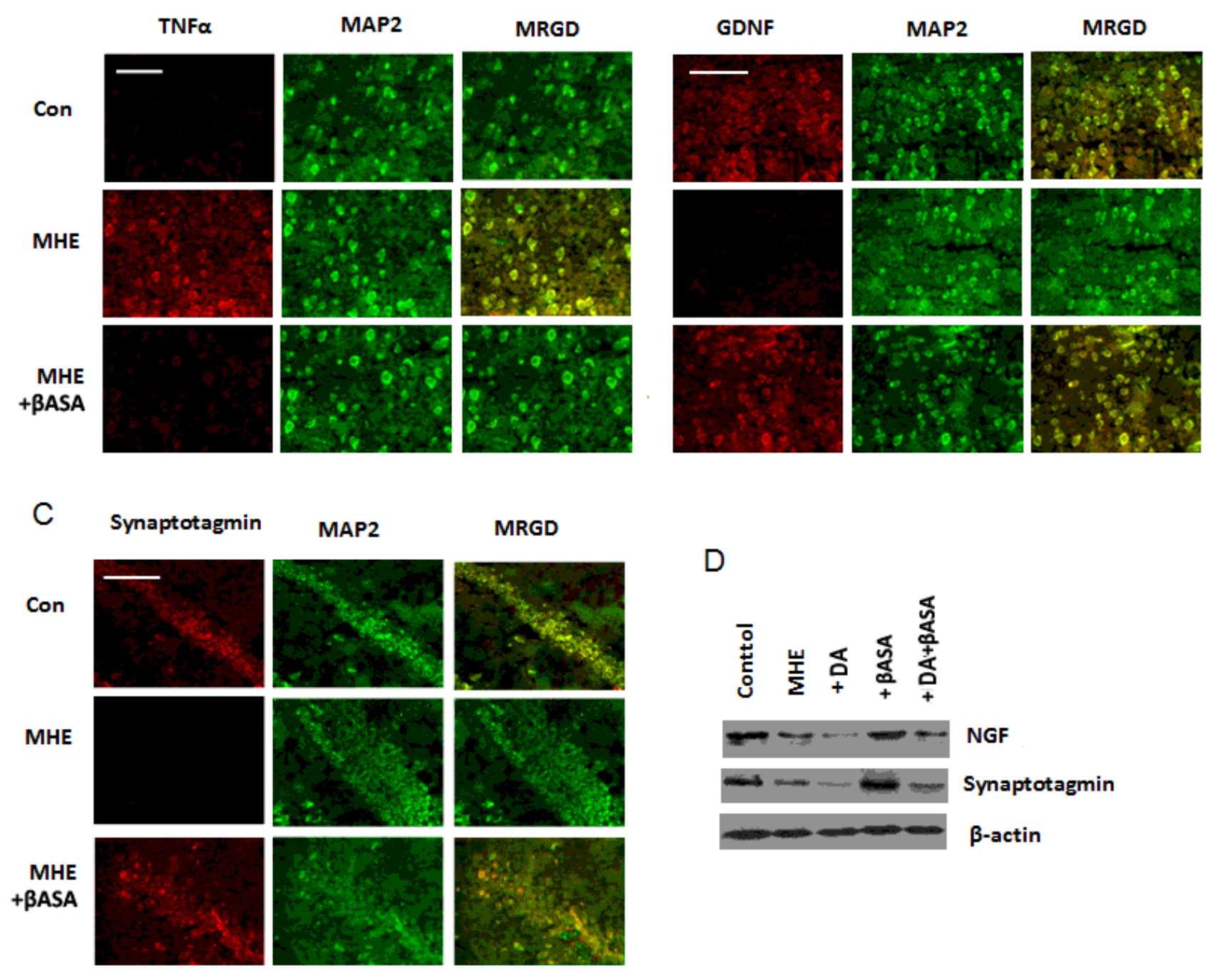

E
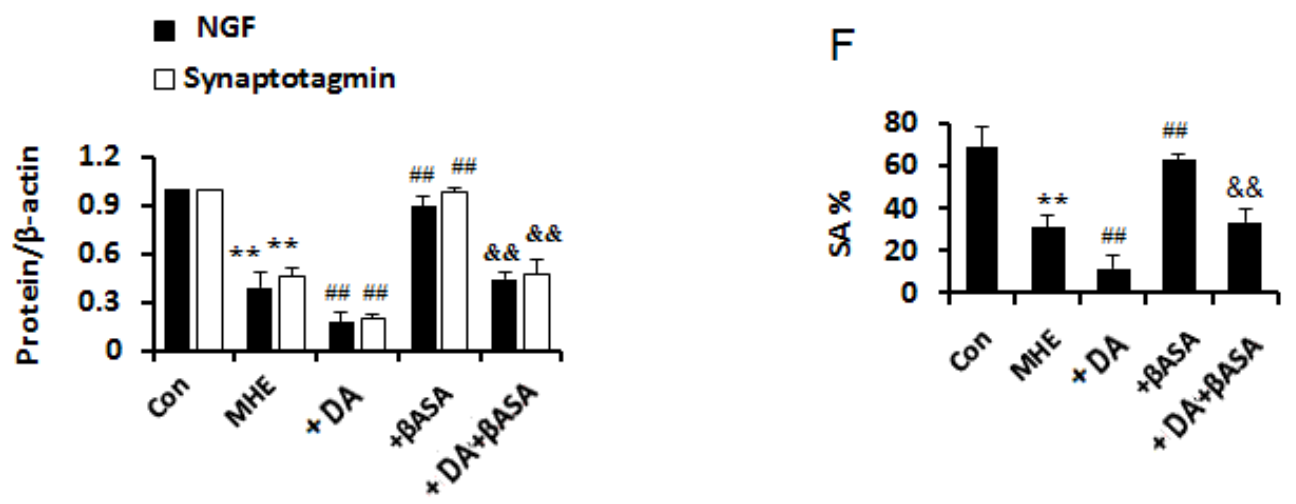

Figure 8

BASA improves inflammation and DA-induced synaptic loss in MHE rats. $(A, B)$ Immunostaining of free floating cortical sections from MHE rats after administration with $B A S A$ using antibodies against TNFa/GDNF (red), GFAP (green). (C) Immunostaining of free floating hippocampal sections from MHE rats after administration with $\beta A S A$ using antibodies against synaptotagmin (red), GFAP (green). (D,E) IB analysis of lysate from MHE rats after administration with DA, ATAB, or DA+ATAB using anti- 
NGF/synaptotagmin and anti- $\beta$-actin antibodies and subsequent densitometry. (F) Spontaneous alternation percentage (SA\%) in YM of MHE rats after administration with DA, ATAB, or DA+ATAB, Data are shown as mean $\pm S D$. ${ }^{*} P<0.05,{ }^{*} \mathrm{P}<0.01$ vs vehicle-treated group. $\# \mathrm{P}<0.05$, \#\#P<0.01 vs control group, \&P <0.05, \&\&P <0.01 vs MHE+DA-treated group. Scr, scrambled. con, control. Scale bar, $25 \mu \mathrm{m}$. MRGD, merged image. 